\title{
Hysteresis Phenomena during Melting of an Ultrathin Lubricant Film
}

\author{
A. V. Khomenko and I. A. Lyashenko \\ Sumy State University, ul. Rimsky-Korsakov 2, Sumy, 40007, Ukraine \\ e-mail: khom@phe.sumdu.edu.ua \\ Received July 26, 2006
}

\begin{abstract}
The influence of a deformational defect of the shear modulus on the melting of an ultrathin lubricant film was investigated in the framework of the Lorenz model used for describing a viscoelastic medium. It was established that the film can undergo both stepwise and continuous melting. Analysis of the lubricant behavior revealed that there are three modes corresponding to a zero shear stress, a Hookean portion in the loading diagram, and a plastic-flow portion. The hysteresis in the dependences of the stationary shear stress on the strain and the friction surface temperature is examined.
\end{abstract}

PACS numbers: 64.60.-i, 62.20.Fe, 62.20.Qp, 68.60.-p

DOI: $10.1134 / \mathrm{S} 1063783407050228$

\section{INTRODUCTION}

In recent years, the problem regarding the sliding friction of smooth solid surfaces with a thin lubricant film in between has attracted considerable attention of researchers [1]. Experimental investigations of the atomically flat mica surfaces separated by an ultrathin lubricant layer have revealed that the lubricant layer under specific conditions exhibits properties of a solid [2]. In particular, Smith et al. [3] and Aranson et al. [4] observed a stick-flip motion specific to dry friction. This boundary mode arises in the case where the thickness of the lubricant film is less than four molecular layers and is explained by the solidification under compression of the walls. Subsequent stepwise melting proceeds when the shear stress exceeds the critical value due to the "shear-induced melting" effect. These films are characterized by the yield stress, which is a characteristic of failure in solids.

In our previous paper [5], we proposed an approach according to which the transition of an ultrathin lubricant film from a solidlike state to a liquidlike state occurs as a result of the thermodynamic and shearinduced melting. A combined analytical description of these processes, which proceed through the self-organization of shear-stress, strain, and temperature fields in the lubricant film, was performed with allowance made for both the additive noises of the above quantities [6, $7]$ and the correlated temperature fluctuations $[8,9]$.

However, the question as to the origin of stepwise melting and the experimentally observed hysteresis [10] remains open. In the present work, the conditions under which these phenomena can be observed were determined by analyzing the deformational defect of the shear modulus in the framework of the Lorenz model used for describing a viscoelastic medium [5]. It was found that there are three stationary modes, namely, two solidlike modes corresponding to dry friction and one liquidlike mode corresponding to sliding friction. It was demonstrated that the crossover from the solidlike mode to the liquidlike mode occurs in accordance with the hysteresis of the dependence of the stress either on the strain (during stepwise melting) or on the temperature (when stepwise melting gives way to a transition mode). Moreover, we analyzed the stability of the transition state and determined the parameters of the system for which the hysteresis takes place.

\section{BASIC EQUATIONS}

In our previous study [5], the system of kinetic equations that govern the mutually consistent behavior of shear stresses $\sigma$ and strains $\varepsilon$, as well as the temperature $T$, in an ultrathin lubricant film undergoing friction between atomically flat mica surfaces was obtained using the rheological description of a viscoelastic medium with thermal conductivity. Let us introduce the units of measurement for the variables $\sigma, \varepsilon$, and $T$ :

$$
\sigma_{s}=\left(\frac{\rho c_{\mathrm{V}} \eta_{0} T_{c}}{\tau_{T}}\right)^{1 / 2}, \quad \varepsilon_{s}=\frac{\sigma_{s}}{G_{0}}, \quad T_{c} .
$$

Here, $\rho$ is the lubricant density, $c_{V}$ is the specific heat capacity, $T_{c}$ is the critical temperature, $\eta_{0} \equiv \eta\left(T=2 T_{c}\right)$ is the characteristic value of the shear viscosity $\eta, \tau_{T} \equiv$ $\rho l^{2} c_{V} / \kappa$ is the thermal conductivity time, $l$ is the thermal conductivity scale, $\kappa$ is the thermal conductivity coefficient, $\tau_{\varepsilon}$ is the strain relaxation time, and $G_{0} \equiv \eta_{0} / \tau_{\varepsilon}$. The aforementioned system of kinetic equations can be written in the form 


$$
\begin{gathered}
\tau_{\sigma} \dot{\sigma}=-\sigma+g(\sigma) \varepsilon, \\
\tau_{\varepsilon} \dot{\varepsilon}=-\varepsilon+(T-1) \sigma, \\
\tau_{T} \dot{T}=\left(T_{e}-T\right)-\sigma \varepsilon+\sigma^{2} .
\end{gathered}
$$

Here, we introduced the stress relaxation time $\tau_{\sigma}$, the temperature $T_{e}$ of atomically flat mica surfaces of friction, and the function $g(\sigma) \equiv G(\sigma) / G_{0}$, where the stressdependent shear modulus of lubricant $G(\sigma)$ is given by the formula

$$
G(\sigma)=\Theta+\frac{G-\Theta}{1+\left(\sigma / \sigma_{p}\right)^{\beta}}, \quad \beta=\text { const }>0 .
$$

At $g(\sigma)=G / G_{0} \equiv$ const, relationship (2) is reduced to a Maxwell-type equation describing a viscoelastic medium via the replacement of $\varepsilon / \tau_{\sigma}$ by $\partial \varepsilon / \partial t$. The Maxwell equation supposes the use of the idealized Hencky model. In this model, the dependence of the stress on the strain $\sigma(\varepsilon)$ is represented by the Hooke law $\sigma=G \varepsilon$ at $\varepsilon<\varepsilon_{m}$ and a constant $\sigma_{m}=G \varepsilon_{m}$ at $\varepsilon \geq \varepsilon_{m}$, where $\sigma_{m}$ and $\varepsilon_{m}$ are the maximum elastic shear stress and the maximum shear strain in the Hookean portion, respectively [at $\varepsilon>\varepsilon_{m}$, there arises a viscous flow with a strain rate $\left.\dot{\varepsilon}=\left(\sigma-\sigma_{m}\right) / \eta\right]$. In actual fact, the dependence $\sigma(\varepsilon)$ involves two portions: the first (Hookean) portion is characterized by a large slope determined by the shear modulus $G$, and the second substantially flatter portion of plastic strain has a slope governed by the hardening coefficient $\Theta<G$. It is clear that such a behavior means that the shear modulus depends on the stress. In order to take this into account, we used the simplest approximation (5), which describes the above crossover from the elastic strain mode to the plastic strain mode. This crossover occurs at characteristic values of shear stresses $\sigma_{p}$ and shear strains $\varepsilon_{p}$. It is should be noted that structural phase transitions of a liquidlike lubricant have been described with the use of third-order invariants, which violate the parity of the dependence of the synergetic potential $V$ on the stress $\sigma$. Therefore, the linear term $\sigma / \sigma_{p}(\beta=1)$ rather than the quadratic term $\left(\sigma / \sigma_{p}\right)^{2}(\beta=2)[11]$ was used in approximation (5) and the corresponding dependence $V(\sigma)$ was not an even function [5].

Expression (3) has the form of the corresponding Kelvin-Voigt equation [5, 12], which has been widely used in the theory of boundary friction and takes into account the dependence of the shear viscosity on the dimensionless temperature $\eta=\eta_{0} /(T-1)$. It should be noted that combination of relationships (2) and (3) represents a new rheological model, because they are reduced to a second-order differential equation with respect to the stress $\sigma$ or the strain $\varepsilon$. Equation (4) is a relationship for the thermal conductivity. This relationship describes the heat transfer from friction surfaces to a lubricant film, the effect of dissipative heating of a viscous liquid flowing under stresses, and the reversible mechanocaloric effect in the linear approximation. Equations (2)-(4) formally coincide with the synergetic Lorenz system $[11,13]$, in which the shear stresses play the role of an order parameter, the conjugate field is reduced to the shear strain, and the temperature is a controlling parameter. As is known, this system has been used for describing both thermodynamic phase and kinetic transformations. It should also be noted that the rheological properties of lubricant films have been experimentally investigated and, hence, the corresponding data allow one to construct phase diagrams [2].

\section{HYSTERESIS BEHAVIOR}

The specific feature of relationship (5) is that it describes a hysteresis upon melting of the thin lubricant layer only in the $\sigma-T_{e}$ coordinates [5]. In this case, the stress-strain curve $\sigma(\varepsilon)$ is monotonic and can represent only a continuous transition. However, experimental data uniquely indicate that melting of molecularly thin lubricant films has a stepwise character [2], although it can occur according to the second-order phase transition mechanism. As will be shown below, the given feature can be described with replacing the dependence $g(\sigma)$ in formula (2) by the dependence $g(\varepsilon) \equiv G(\varepsilon) / G_{0}$, where

$$
G(\varepsilon)=\Theta+\frac{G-\Theta}{1+\left(\varepsilon / \varepsilon_{p}\right)^{\beta}} .
$$

It should be noted that the parameter $\beta>0$ determining the potential behavior plays a key role.

Let us consider a stationary state in which the derivative in relationship (2) is equal to zero $(\dot{\sigma}=0)$ and the stress $\sigma$ in the lubricant remains constant. In this case, we obtain an expression similar to the relationship describing the Hooke law; that is,

$$
\begin{gathered}
\sigma=g(\varepsilon) \varepsilon, \\
g(\varepsilon)=g_{\theta}\left(1+\frac{\theta^{-1}-1}{1+(\varepsilon / \alpha)^{\beta}}\right),
\end{gathered}
$$

where we introduced the parameter $\theta=\Theta / G<1$ determined by the ratio between the slopes of the Hookean and plastic portions in the stress-strain curve and the coefficients $g_{\theta}=\Theta / G_{0}<1$ and $\alpha=\varepsilon_{p} / \varepsilon_{s}$. The dependence constructed using expression (7) at specific quantities $\alpha, g_{\theta}$, and $\theta$ is depicted in Fig. 1 . It can be seen from this figure that two situations are possible: the dependence $\sigma(\varepsilon)$ is represented by an ascending monotonic curve at small parameters $\beta$ (inset to Fig. 1) and becomes nonmonotonic at parameters

$$
\beta>\frac{1+\sqrt{\theta}}{1-\sqrt{\theta}} .
$$

The lubricant undergoes continuous melting in the former case and stepwise melting in the latter case when the stress increases to the point $A$ and the system 


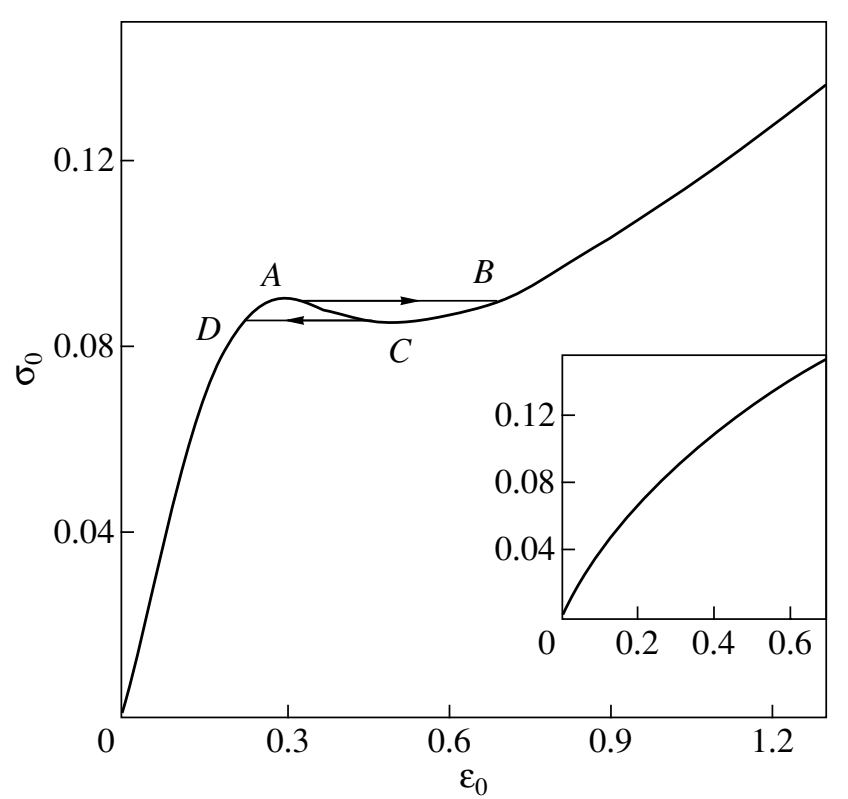

Fig. 1. Dependence of the stationary shear stress $\sigma_{0}$ on the strain $\varepsilon_{0}$ [expression (7)] for parameters $\theta=0.2, g_{\theta}=0.1$, $\alpha=0.3$, and $\beta=3.0$. The inset shows the corresponding dependence for $\beta=1.0$.

goes into the point $B$. A further increase in the stress is accompanied by a monotonic increase in the strain and the lubricant remains in a liquidlike state. As the stress decreases, the lubricant to the point $C$ retains a liquidlike structure and then abruptly transforms into a solidlike state and the system goes into the point $D$. With a further decrease in the stress, the lubricant remains in the solidlike state. Similar transitions are represented as first-order phase transitions [14] between states that are not true thermodynamic phases. These transformations can be explained within the concept of shear melting [4]. It should be noted that the described hysteresis behavior was observed experimentally $[10,15]$.

By using expression (7), we can determine the abscissas of the transition points $A$ and $C$. As a result, it becomes clear that the strain jump upon melting increases with an increase in the coefficient $\alpha$ and that an increase in the parameter $\beta$ leads to $\varepsilon_{A}-\varepsilon_{C} \longrightarrow 0$. Therefore, at large parameters $\beta$ (small coefficients $\alpha$ ), melting and solidification occur virtually at the same strain $\left(\varepsilon_{A} \approx \varepsilon_{C}\right)$ but at different stresses $\sigma$. As in our earlier works [5-9], the shear stress will serve as the order parameter: the lubricant is in a liquidlike state at $\sigma>\sigma_{A}$ and in a solidlike state at $\sigma<\sigma_{C}$. In the intermediate range $\sigma_{C}<\sigma<\sigma_{A}$, the lubricant is unstable and can exist in both states.

The dependence of the stationary shear stress $\sigma_{0}$ on the friction surface temperature $T_{e}$ is plotted in Fig. 2. Below the critical temperature $T_{c 0}$, the stresses in the lubricant are absent $(\sigma=0)$ and the lubricant is in the solidlike state. An increase in the temperature to the

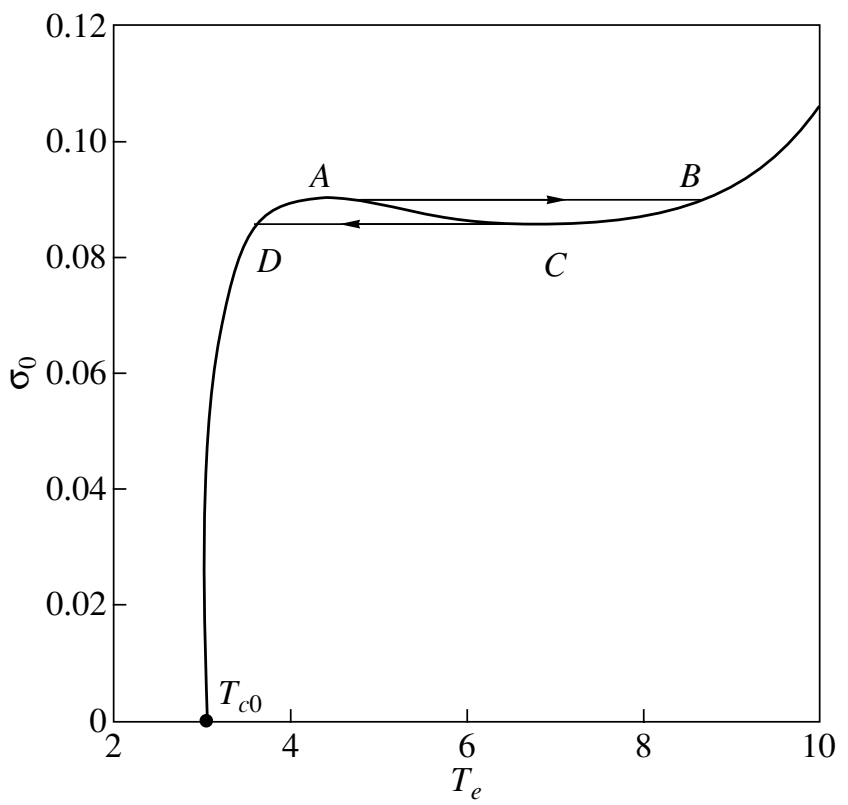

Fig. 2. Dependence of the stationary shear stress $\sigma_{0}$ on the friction surface temperature $T_{e}$. The parameters are the same as in Fig. 1.

point $A$ results in an increase in the stress, which corresponds to the Hookean elastic portion in the stressstrain curve depicted in Fig. 1, and, hence, the lubricant remains in the solidlike state. With a further increase in the temperature in the range $T_{c A}<T_{e}<T_{c B}$ (where the temperatures $T_{c A}$ and $T_{c B}$ corresponds to the points $A$ and $B$, respectively), the stress remains constant and the lubricant retains its structure. At temperatures above $T_{c B}$, the stress-strain dependence exhibits a plastic behavior (see Fig. 1) and the lubricant transforms into a liquidlike state. This corresponds to a liquid friction. As the surface friction temperature decreases, the lubricant remains in the liquidlike state to the temperature $T_{c C}$, then the stress remains unchanged to $T_{e}=T_{c D}$, and the lubricant has a solidlike structure at temperatures below $T_{c D}$. The relationship for the critical temperature $T_{c 0}$ can be obtained from the condition $\partial V / \partial \sigma=0$ [where $V$ is the synergetic potential defined by formula (13)]; that is,

$$
T_{c 0}=1+\theta / g_{\theta} \equiv 1+G_{0} / G .
$$

In the temperature range $T_{c D}<T_{e}<T_{c B}$, the lubricant can be both in liquid- and solidlike states depending on the prehistory of the system. In the range $T_{c A}<T_{e}<T_{c C}$, which is covered by the aforementioned temperature range, the stationary stress remains constant. Most likely, the equality $\sigma_{0}=$ const is satisfied with an increase in the temperature in the range $T_{c A}<T_{e}<T_{c B}$, because the energy should be transferred to the lubricant for melting. The stress remains unchanged in the range $T_{c D}<T_{e}<T_{c C}$ with a decrease in the friction sur- 
face temperature, because the lubricant should lose energy in order to transform into the solidlike state. It is believed that solidlike structures of the lubricant at temperatures below and above the point $T_{c 0}$ should differ from each other, since the transition between them proceeds through the second-order phase transition mechanism (Fig. 2).

In the adiabatic approximation $\tau_{\varepsilon} \ll \tau_{\sigma}$ and $\tau_{T} \ll \tau_{\sigma}$ [5], we can set $\tau_{\varepsilon} \dot{\varepsilon} \approx 0$ and $\tau_{T} \dot{T} \approx 0$. As a result, from relationships (3) and (4), we find

$$
\begin{aligned}
& \varepsilon=\sigma-\left(2-T_{e}\right) \frac{\sigma}{1+\sigma^{2}}, \\
& T=T_{e}+\left(2-T_{e}\right) \frac{\sigma^{2}}{1+\sigma^{2}} .
\end{aligned}
$$

Substitution of expression (10) into formula (2) gives the Landau-Khalatnikov equation

$$
\tau_{\sigma} \dot{\sigma}=-\frac{\partial V}{\partial \sigma}
$$

with the synergetic potential

$$
V=\frac{\sigma^{2}}{2}-\int_{0}^{\sigma} g(\varepsilon) \varepsilon d \sigma,
$$

where the dependence $\varepsilon(\sigma)$ is given by relationship (10). It should be noted that, in the temperature range $T_{c A}<$ $T_{e}<T_{c C}$ this potential does not lead to correct results, because the model has an unstable solution that corresponds to a decrease in the stress with an increase in the strain and does include the hysteresis. In order to determine the potential form in the aforementioned temperature range, relationship (2) is replaced by the equation

$$
\tau_{\sigma} \dot{\sigma}=-\sigma+\delta,
$$

where $\delta$ is a constant stress. In the Landau-Khalatnikov equation (12), the potential is defined by the expression

$$
V=\frac{\sigma^{2}}{2}-\delta \sigma
$$

It can be seen that the potential depends only on the stress $\sigma$; i.e., in the situation where the stress is retained with a change in the temperature, the potential $V$ remains unchanged.

Figure $3 \mathrm{a}$ depicts the dependences of the synergetic potential on the shear stress at constant friction surface temperatures [according to the calculations with the use of expression (13)]. Curve 1 corresponds to a temperature below the critical point $T_{c 0}$. As can be seen from Fig. 3a, this curve exhibits one zero minimum, the stress in the lubricant is absent, and, hence, the lubricant is in a solidlike state. Curve 2 is calculated at a temperature in the range $T_{c 0}<T_{e}<T_{c D}$ and contains a nonzero minimum, which corresponds to the Hookean
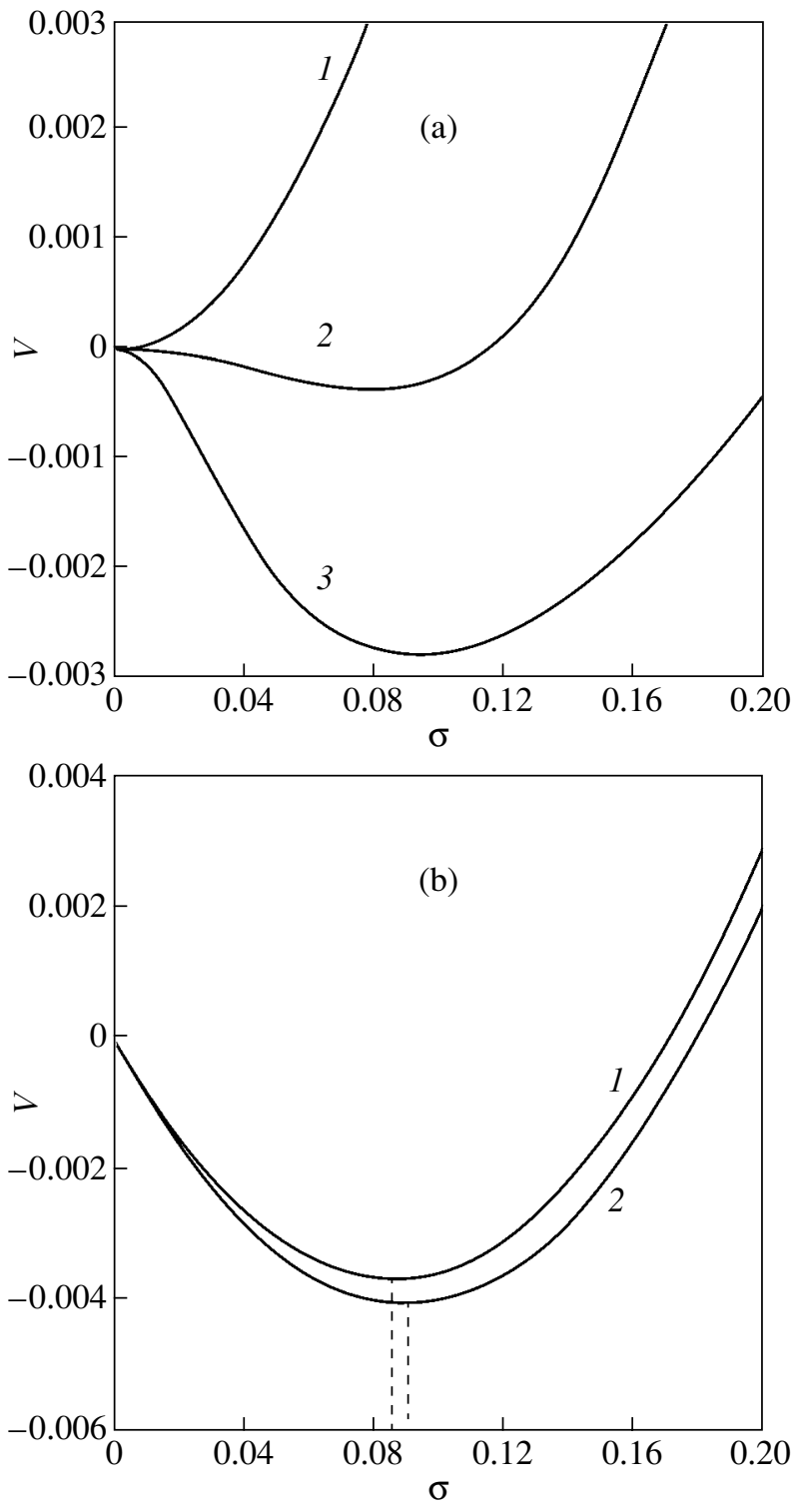

Fig. 3. Dependences of the synergetic potential $V$ on the shear stress $\sigma$ according to the calculations with the use of (a) relationship (13) for temperatures $T_{e}=$ (1) 1.0, (2) 3.4, and (3) 9.0 and (b) relationship (15) for the cases of (1) solidification and (2) melting. The parameters are the same as in Fig. 2.

portion in Fig. 1. This implies that the lubricant is also in a solidlike state. Curve 3 is constructed at a temperature $T_{e}>T_{c B}$ and is characterized by one nonzero minimum. Since the system under these conditions corresponds to the plastic-flow portion, the lubricant is in a liquidlike state.

The transition between the first and second states proceeds through the second-order phase transition mechanism, because the zero minimum transforms into a nonzero minimum. Since curves 2 and 3 are identical in shape, the crossover between the corresponding friction modes should be interpreted as a kinetic transfor- 
mation rather than a phase transformation [14]. Therefore, the solidlike structure of the lubricant at temperatures below the critical point $T_{c 0}$ is similar to the solid state, whereas the solidlike structure at temperatures above this point bears a resemblance to the liquid state, but, on the whole, the lubricant is characterized by a solidlike behavior. A further crossover to a liquid friction mode is attended by a decrease in the viscosity of the lubricant, which begins to flow.

It should be noted that, in the range where the stress remains constant between the solidlike and liquidlike phases, the lubricant is in an intermediate state and has a structure that differs from the structures of these phases. The dependence of the synergetic potential calculated from expression (15) on the stress for the intermediate state is plotted in Fig. 3b. Curves 1 and 2 correspond to the straight lines $C D$ (solidification) and $A B$ (melting) in Fig. 2, respectively. It can be seen from Fig. $3 b$ that the minimum of the potential upon transition to the solidlike structure is located higher than the corresponding minimum upon transition to the liquidlike state. This means that the latter process is more stable. Since both states in the temperature range $T_{c A}<$ $T_{e}<T_{c C}$ can coexist at the same temperature $T_{e}$, transitions can occur between these (unstable) phases. These transitions lead to a stick-slip friction [16]. However, consideration of this regime is beyond the scope of the present work. As was shown above, the process represented by the straight line $A B$ is more stable; i.e., there exists a higher probability that the lubricant upon crossover between different friction modes is in the state corresponding to this straight line. The potentials corresponding to the temperature ranges $T_{c D}<T_{e}<T_{c A}$ and $T_{c C}<T_{e}<T_{c B}$, in the which the stress varies, are similar to those represented by curves 2 and 3 in Fig. 3a, respectively. It can be seen from Fig. $3 b$ that, in these ranges, the potential well in the case where the stress remains constant is lower than the potential well in the case where the stress varies as is shown in Fig. 2. Therefore, the transition state is more stable and the transformation proceeds via the aforementioned path according to the hysteresis. However, three friction modes can correspond to the same temperature in the $A C$ region: two transition modes and one unstable mode, which directly follows from the model under consideration. The instability of the last mode is associated with the fact that, in this state, first, the potential is higher than the potentials in both transition states and, second, the stress decreases with an increase in the strain.

\section{CONCLUSIONS}

Thus, the above analysis demonstrated that the hysteresis observed upon melting of a thin lubricant film can be described with allowance made for the deformational defect of the shear modulus. The main feature of the lubricant behavior is that the lubricant melts and solidifies at different values of the shear stress, which plays the role of an order parameter. It was revealed that the lubricant can be in two solidlike and one liquidlike states and that the hysteresis is observed upon phase transition between these states. The stability of the transition states upon melting and solidification was analyzed.

\section{REFERENCES}

1. B. N. J. Persson, Sliding Friction: Physical Principles and Applications (Springer, Berlin, 1998).

2. H. Yoshizawa, Y.-L. Chen, and J. Israelachvili, J. Phys. Chem. 97, 4128 (1993); H. Yoshizawa and J. Israelachvili, J. Phys. Chem. 97, 11300 (1993).

3. E. D. Smith, M. O. Robbins, and M. Cieplak, Phys. Rev. B: Condens. Matter 54, 8252 (1996).

4. I. S. Aranson, L. S. Tsimring, and V. M. Vinokur, Phys. Rev. B: Condens. Matter 65, 125402 (2002).

5. A. V. Khomenko and O. V. Yushchenko, Phys. Rev. E: Stat., Nonlinear, Soft Matter Phys. 68, 036110 (2003).

6. A. V. Khomenko, Phys. Lett. A 329, 140 (2004).

7. A. V. Khomenko and I. A. Lyashenko, Zh. Tekh. Fiz. 75 (11), 17 (2005) [Tech. Phys. 50 (11), 1408 (2005)].

8. A. V. Khomenko, Vestn. Sumsk. Gos. Univ. (Ukr.), No. 10, 15 (2004).

9. A. V. Khomenko and Ya. A. Lyashenko, Vestn. Sumsk. Gos. Univ. (Ukr.), No. 4, 70 (2005).

10. A. L. Demirel and S. Granik, J. Chem. Phys. 109, 6889 (1998).

11. A. I. Olemskoŭ and A. V. Khomenko, Zh. Éksp. Teor. Fiz. 110, 2144 (1996) [JETP 83, 1180 (1996)].

12. Rheology: Theory and Applications, Ed. by F. R. Eirich (Academic, New York, 1956; Inostrannaya Literatura, Moscow, 1962).

13. H. Haken, Synergetics (Springer, Heidelberg, 1978; Mir, Moscow, 1980).

14. E. A. Brener and V. I. Marchenko, Pis'ma Zh. Éksp. Teor. Fiz. 76 (4), 246 (2002) [JETP Lett. 76 (4), 211 (2002)].

15. V. L. Popov, Zh. Tekh. Fiz. 71 (5), 100 (2001) [Tech. Phys. 46 (5), 605 (2001)].

16. L. B. Zuev and V. I. Danilov, Philos. Mag. A 79, 43 (1999).

Translated by O. Borovik-Romanova 